\title{
Factors associated with progression to infection in methicillin- resistant Staphylococcus aureus-colonized, critically ill neonates
}

\author{
Carly R. Schuetz $\mathbb{1}^{1} \cdot$ Patrick G. Hogan ${ }^{1}$ Patrick J. Reich ${ }^{1} \cdot$ Sara Halili ${ }^{1} \cdot$ Hannah E. Wiseman ${ }^{1} \cdot$ Mary G. Boyle ${ }^{1} \cdot$ \\ Ryley M. Thompson ${ }^{1} \cdot$ Barbara B. Warner $\mathbb{D}^{1} \cdot$ Stephanie A. Fritz $\mathbb{D}^{1}$
}

Received: 30 July 2020 / Revised: 23 November 2020 / Accepted: 19 January 2021 / Published online: 1 March 2021

(c) The Author(s), under exclusive licence to Springer Nature America, Inc. 2021

\begin{abstract}
Objective To identify factors associated with development of symptomatic infection in infants colonized with methicillinresistant Staphylococcus aureus (MRSA) in the Neonatal Intensive Care Unit (NICU).

Study design This case-control study was performed at St. Louis Children's Hospital NICU from 2009 to 2019. The MRSA-colonized infants who developed symptomatic MRSA infection (cases) were matched 1:3 with MRSA-colonized infants who did not develop infection (controls). Demographics and characteristics of NICU course were compared between groups. Longitudinal information from subsequent hospitalizations was also obtained.

Results Forty-two infected cases were compared with 126 colonized-only controls. Cases became colonized earlier in their NICU stay, were less likely to have received mupirocin for decolonization, and had a longer course of mechanical ventilation than controls. Longitudinally, cases had a more protracted NICU course and were more likely to require hospital readmission. Conclusion Progression from MRSA colonization to symptomatic infection is associated with increased morbidity and may be mitigated through decolonization.
\end{abstract}

\section{Introduction}

In hospitalized neonates, Staphylococcus aureus is a common cause of late-onset infection, leading to significant morbidity, mortality, and prolonged hospitalization in those who are infected [1]. Methicillin-resistant $S$. aureus (MRSA) infections in neonates include skin and soft tissue infections, respiratory tract infections, pneumonia, bacteremia, osteomyelitis, and septic arthritis [2]. Morbidities that occur as a result of late-onset infection include a prolonged need for mechanical ventilation and subsequent bronchopulmonary dysplasia, necessitating longer hospital courses and decreasing likelihood of survival [1]. A study in a Neonatal Intensive Care Unit (NICU) in North Carolina estimated daily hospitalization costs between $\$ 2000$ and $\$ 5000$, depending on gestational age [3]. A prolonged hospital course secondary to $S$. aureus infection can thus

Stephanie A. Fritz

fritz.s@wustl.edu

1 Department of Pediatrics, Washington University School of Medicine, St. Louis, MO, US exponentiate costs for both families and hospital systems. A 2010 study performed in Washington, D.C. demonstrated a 40 days increase in NICU hospitalization for infants with MRSA infection, associated with a greater than $\$ 160,000$ increase in cost incurred by the family [4].

MRSA colonization is a demonstrated risk factor for MRSA infection [5]. According to a recent meta-analysis, MRSA-colonized neonates were 24 times more likely to develop an MRSA infection than noncolonized neonates [6]. The prevalence of MRSA nasal colonization in neonates varies, typically between $2 \%$ and $4 \%$ [6-9], increasing to as high as $8 \%$ during an MRSA outbreak investigation [10]. Infants in the NICU are at risk for MRSA colonization secondary to transmission from colonized parents, healthcare workers, and contaminated hospital surfaces [2]. Factors associated with MRSA colonization in the NICU include lower birth weight, younger gestational age, invasive procedures, and indwelling catheters [2]. In an effort to prevent infection, decolonization with topical antimicrobials (e.g., mupirocin and chlorhexidine) is frequently employed for colonized neonates [11, 12].

Neonates colonized with MRSA are at greater risk of MRSA infection than those who are not colonized [13-15]. However, only a subset of colonized infants develop 
symptomatic infection. The objective of this study was to identify factors associated with, and longitudinal outcomes related to, development of symptomatic MRSA infection in MRSA-colonized infants in the NICU.

\section{Methods}

\section{Setting}

This case-control study employed data from patients hospitalized between July 2009 and June 2018 in the St. Louis Children's Hospital (SLCH) NICU, a level IV NICU adjacent to the Labor and Delivery Unit at Barnes-Jewish Hospital. The SLCH NICU has 85 beds (increased to 125 beds in February 2018) and $\approx 1300$ admissions per year.

An MRSA active surveillance program was implemented in the SLCH NICU in 2004. All neonates admitted to the SLCH NICU were screened for MRSA colonization (via anterior nasal swab) at admission and weekly throughout their NICU hospitalization. Infants with positive MRSA surveillance cultures were placed in contact isolation, requiring healthcare workers to wear gowns and gloves when handling the infants for the remainder of their hospitalization. A standard decolonization protocol was introduced in 2006 for MRSA-colonized neonates consisting of intranasal $2 \%$ mupirocin ointment applied twice daily for 7 days and, at $>30$ weeks of gestation, a one-time bath from the neck down with $2 \%$ chlorhexidine gluconate cloths.

\section{Study population}

Infants were selected from a hospital-generated database of MRSA-colonized infants detected through active surveillance. MRSA-colonized infants who developed symptomatic infection (cases, "infected") were matched 1:3 to MRSA-colonized infants with overlapping NICU courses who did not develop infection (controls, "colonized-only").

Between July 2009 and June 2018, there were 7501 admissions to the SLCH NICU. Over that time, 325 infants were colonized with MRSA (4\%) (Fig. 1); 62 patients (19\% of those colonized) developed symptomatic MRSA infection. Infants with exposure outside of a NICU setting were excluded from the study, including infants who had been discharged from a hospital to home prior to admission to SLCH NICU $(n=1)$ and those hospitalized in another SLCH unit prior to NICU transfer $(n=4)$. Infants who spent $>1$ month at an outside hospital prior to SLCH NICU admission $(n=8)$ and infants for whom MRSA colonization was first detected $>1$ week after development of symptomatic infection $(n=7)$ were also excluded.

Symptomatic MRSA infection was defined as a bodily fluid culture-positive for MRSA (e.g., bacteremia, skin/soft

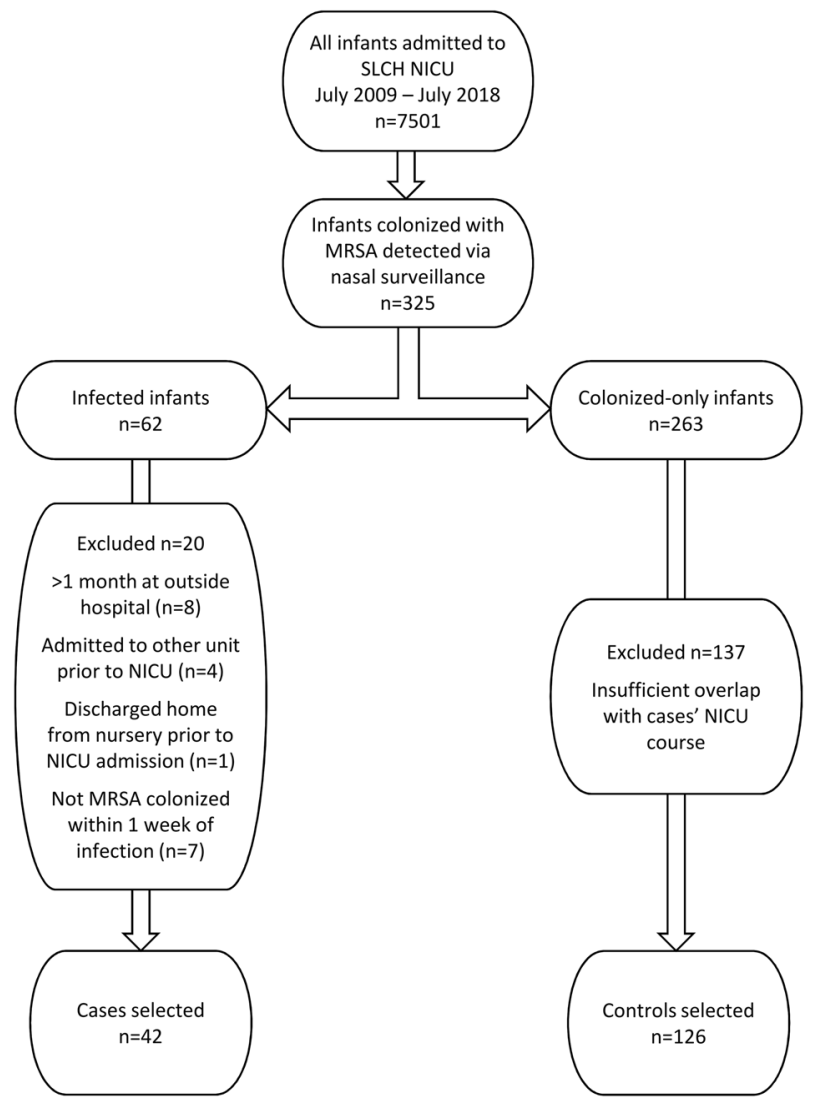

Fig. 1 Assembly of study cohort. MRSA-colonized infants who developed symptomatic infection (cases; infected) were matched 1:3 to MRSA-colonized infants with overlapping NICU courses who did not develop infection (controls; colonized-only).

tissue infection, urinary tract infection, and conjunctivitis). Patients with tracheitis, defined as a tracheal aspirate culture yielding MRSA in the presence of polymorphonuclear leukocytes on Gram stain, who required escalating respiratory support and/or demonstrated other signs of clinical instability and were treated with antibiotics, were also categorized as having symptomatic infection [16].

The study included three sets of twins (in one set, both twins were infected cases; in two sets, one twin was an infected case and the other twin was a colonized-only control) and one set of quadruplets (one infected case, three colonized-only controls). Data were abstracted for 162 mothers of the enrolled neonates. This study was approved by the Washington University School of Medicine Institutional Review Board with waiver of consent for the infants and mothers.

\section{Data collection}

Data were abstracted from electronic medical records to obtain neonates' demographic data and characteristics of hospital stay, including sex, race, gestational age, birth weight, birth location (born at our medical center [inborn] 
vs. born at an outside institution [outborn]), mode of delivery, presence of multiple gestations, comorbidities, surgical procedures, nutrition via nasogastric or orogastric tube, endotracheal intubation, number of ventilator days, presence of a central line and number of central line days, type of antibiotics received and number of systemic antibiotic days, number of systemic steroid days, exposure to maternal or donor breast milk, results of all MRSA surveillance cultures, results of all bodily fluid cultures, and receipt of intranasal mupirocin for decolonization following detection of MRSA colonization. Documentation of chlorhexidine gluconate baths was not readily available for this cohort. Maternal data obtained included race, previous MRSA-positive cultures, and presence of antepartum antibiotics.

Post-discharge longitudinal data were also obtained for neonates, when available. Information obtained included follow-up visits in newborn medicine clinic, medical diagnoses carried, subsequent hospitalizations, and subsequent surveillance and bodily fluid cultures obtained.

\section{Statistical analyses}

Statistical analyses were performed with SPSS for Windows v26 (IBM SPSS, Chicago, IL). For infected infants, data collected and presented represent characteristics of NICU course prior to infection to determine factors associated with developing an infection; these characteristics included mupirocin for decolonization, surgical procedures, nasogastric or orogastric tube, maternal breast milk, donor breast milk, intubation, ventilator days, central venous access, central line days, antibiotics, antibiotic days, inotropes, steroids, and steroid days. MRSA-colonized neonates who did and did not develop infection were compared using Mann-Whitney $U$ test (continuous data) and Fisher's exact test (categorical data). All tests of significance were twotailed and $p$ values $<0.05$ were considered statistically significant. To determine the factors independently associated with development of symptomatic infection, all variables tested in univariate analysis were then evaluated in a backward stepwise multivariable logistic regression. Longitudinal data analysis compared the likelihood of readmission between infected and colonized-only infants. The factors associated with hospital readmission after NICU discharge were also assessed using multivariable logistic regression; in this model, the characteristics of entire NICU course, not just prior to infection, were included.

\section{Results}

The cohort was comprised of 168 neonates: 42 MRSAcolonized infants who developed symptomatic MRSA infection (cases) and 126 MRSA-colonized infants who did not develop infection (controls). The 42 infected infants most commonly experienced tracheitis $(n=22)$, followed by infection of multiple sites (bacteremia plus tracheitis $[n=6]$, bacteremia plus skin abscess $[n=1])$, bacteremia $(n=6)$, skin and soft tissue infection $(n=5)$, conjunctivitis $(n=1)$, and UTI $(n=1)$. Median time from admission to detection of colonization was 18 days (interquartile range [IQR] 10-30 days); median time from admission to infection was 24 days (IQR 14-43 days). Median time from detection of colonization to infection was 4 days (IQR 1-11 days).

\section{Factors associated with MRSA infection}

Infected infants had a lower median gestational age of 26 weeks, 1 day (IQR 24 weeks, 3 days-32 weeks, 6 days) than the colonized-only infants, with median gestational age 29 weeks, 6 days (IQR 27 weeks, 5 days-32 weeks, 5 days, $p=0.002$, Table 1). Concordantly, infected infants had a lower median birth weight of $915 \mathrm{~g}$ (IQR $725 \mathrm{~g}-1668 \mathrm{~g}$ ) than colonized-only infants, with median birth weight 1320 $\mathrm{g}$ (IQR $925 \mathrm{~g}-1913 \mathrm{~g}, p=0.004)$. The proportions of male (30 [71\%] infected, 76 [60\%] colonized-only) and inborn infants (28 [67\%] infected, 90 [71\%] colonized-only) were similar between groups. Median time for detection of colonization (15 days for infected infants, 19 days for colonized-only infants) was also similar.

The proportion of intubated neonates was similar between groups (Table 1); however, infected infants were exposed to longer courses of mechanical ventilation (median 15 days [IQR 4-25 days]) than colonized-only infants (median 2 days [IQR $1-10$ days], $p<0.001$ ). Most infants in both groups required central vascular access (e.g., umbilical catheters, PICC lines), though infected infants had a greater number of central line days (median 16 days [IQR 12-27 days]) than colonized-only infants (median 13 days [IQR 7-21 days], $p=0.009$ ).

Most infants in both groups (40 [95\%] infected, 123 [98\%] colonized-only) required a nasogastric or orogastric tube (Table 1). Thirty-four ( $81 \%$ ) infected infants received maternal breast milk compared to $114(91 \%)$ colonized-only infants $(p=0.11)$; infected infants were more likely to receive donor breast milk (18 [43\%]) than colonized-only infants (18 [14\%], $p<0.001)$. Infected infants (19 [45\%]) were less likely to have received mupirocin for decolonization following detection of colonization than colonizedonly infants (115 [91\%], $p<0.001)$.

Prior to developing infection, 37 (88\%) infected infants received antibiotics (16 [38\%] received antibiotics with activity against MRSA); 111 (88\%) colonized-only infants received antibiotics for sepsis evaluation or non-MRSA infection (50 [40\%] with activity against MRSA) (Table 1). 
Table 1 Characteristics of Neonatal Intensive Care Unit course for neonates with methicillin-resistant Staphylococcus aureus colonization.

\begin{tabular}{|c|c|c|c|c|c|}
\hline Factor & $\begin{array}{l}\text { Total } \\
n=168(\%)\end{array}$ & $\begin{array}{l}\text { Infected } \\
n=42(\%)\end{array}$ & $\begin{array}{l}\text { Colonized-only } \\
n=126(\%)\end{array}$ & $P$ value $^{\mathrm{a}}$ & $\mathrm{aOR}(95 \% \mathrm{CI})^{\mathrm{b}}$ \\
\hline Gestational age, weeks.days, median (IQR) & $29.5(26.3-32.6)$ & $26.1(24.3-32.6)$ & $29.6(27.5-32.5)$ & 0.002 & - \\
\hline Birth weight, grams, median (IQR) & $1210(843-1869)$ & $915(725-1668)$ & $1320(925-1913)$ & 0.004 & - \\
\hline Male & $106(63)$ & $30(71)$ & $76(60)$ & 0.27 & - \\
\hline Race & & & & & - \\
\hline White & $93(55)$ & $21(50)$ & $72(57)$ & 0.48 & \\
\hline Black and other ${ }^{c}$ & $75(45)$ & $21(50)$ & $54(43)$ & & \\
\hline Caesarean delivery (vs. vaginal) & $118(70)$ & $29(69)$ & $89(71)$ & 0.85 & - \\
\hline Inborn & $118(70)$ & $28(67)$ & $90(71)$ & 0.56 & - \\
\hline $\begin{array}{l}\text { Days from admission to colonization, } \\
\text { median (IQR) }\end{array}$ & $18(10-30)$ & $15(11-26)$ & $19(10-39)$ & 0.34 & $0.97(0.94-0.99)$ \\
\hline $\begin{array}{l}\text { Received mupirocin for MRSA } \\
\text { decolonization }\end{array}$ & $134(80)$ & $19(45)$ & $115(91)$ & $<0.001$ & $0.06(0.02-0.19)$ \\
\hline Surgical procedures ${ }^{\mathrm{d}}$ & $51(30)$ & $14(33)$ & $37(29)$ & 0.70 & - \\
\hline Nasogastric or orogastric tube $\mathrm{d}^{\mathrm{d}}$ & $163(97)$ & $40(95)$ & $123(98)$ & 0.60 & - \\
\hline Received maternal breast milk ${ }^{\mathrm{d}}$ & $148(88)$ & $34(81)$ & $114(91)$ & 0.11 & - \\
\hline Received donor breast milk ${ }^{\mathrm{d}}$ & $36(21)$ & $18(43)$ & $18(14)$ & $<0.001$ & $7.73(2.53-23.68)$ \\
\hline Intubated $^{\mathrm{d}}$ & $141(84)$ & $39(93)$ & $102(81)$ & 0.09 & - \\
\hline Ventilator days $^{\mathrm{d}}$, median (IQR) & $2(1-15)$ & $15(4-25)$ & $2(1-10)$ & $<0.001$ & $1.09(1.04-1.14)$ \\
\hline Central venous access ${ }^{\mathrm{d}}$ & $152(91)$ & $40(95)$ & $112(89)$ & 0.36 & - \\
\hline Central line days ${ }^{\mathrm{d}}$, median (IQR) & $14(8-22)$ & $16(12-27)$ & $13(7-21)$ & 0.009 & - \\
\hline Received antibiotics $^{\mathrm{d}}$ & $148(88)$ & $37(88)$ & $111(88)$ & 1.0 & - \\
\hline Antibiotic days ${ }^{\mathrm{d}}$, median (IQR) & $6(3-11)$ & $7(3-13)$ & $5(3-11)$ & 0.26 & $0.91(0.85-0.98)$ \\
\hline Received inotropes $^{\mathrm{d}}$ & $36(21)$ & $13(31)$ & $23(18)$ & 0.09 & - \\
\hline Received steroids ${ }^{\mathrm{d}}$ & $48(29)$ & $17(41)$ & $31(25)$ & 0.08 & - \\
\hline Steroid days ${ }^{\mathrm{d}}$, median $(\mathrm{IQR})$ & $0(0-2)$ & $0(0-3)$ & $0(0-0.25)$ & 0.12 & - \\
\hline
\end{tabular}

aOR adjusted odds ratio, CI confidence interval, IQR interquartile range, MRSA methicillin-resistant Staphylococcus aureus.

${ }^{a}$ Univariate analysis by Mann-Whitney $U$ test (continuous data) and Fisher's exact test (categorical data).

'Multivariable analysis by backwards stepwise logistic regression: aOR and 95\% CI presented for variables remaining in final model; "—”" represent variables not retained in final model.

${ }^{\mathrm{c}}$ Other race includes biracial $(n=8)$ and Asian $(n=3)$.

${ }^{\mathrm{d}}$ Data collected and presented for infected infants represent characteristics of NICU course prior to infection to determine factors associated with development of an infection.

The number of antibiotic days was similar between groups: median 7 days for infected infants (for the time period prior to infection; IQR 3-13 days) and 5 days for colonized-only infants (over their entire NICU course; IQR 3-11 days, $p=$ 0.26). All infected infants then received systemic antibiotics to treat their MRSA infection. Thirteen (31\%) infected infants received inotropes prior to developing infection compared to $23(18 \%)$ colonized-only infants ( $p=0.09) ; 17$ $(41 \%)$ infected infants received systemic steroids prior to developing infection compared to $31(25 \%)$ colonized-only infants $(p=0.08)$.

In multivariable analysis, infants who developed symptomatic MRSA infection were less likely to have received mupirocin for decolonization (adjusted odds ratio [aOR] $0.06,95 \%$ confidence interval $[\mathrm{CI}] 0.02-0.19$ ), became colonized earlier in their NICU stay (aOR 0.97, 95\% CI 0.94-0.99), and received fewer days of systemic antibiotics (aOR 0.91, 95\% CI 0.85-0.98) than infants who did not develop infection. Infected infants were more likely to have received donor breast milk (aOR 7.73, 95\% CI 2.53-23.68) and had a more prolonged course of mechanical ventilation (aOR 1.09, 95\% CI 1.04-1.14) than infants who did not develop infection.

\section{Morbidities in patients with MRSA infection}

Overall, MRSA-infected infants were exposed to longer courses of antibiotics and steroids during their hospitalization compared to colonized-only infants. Over the entire NICU course, infected infants received a median of 27 days (IQR 
16-46) of systemic antibiotics and 33 days (of 29 infants that received steroids; IQR 7-68 days) of systemic steroids. Of 42 MRSA-infected infants, $14(33 \%)$ required at least one surgical procedure prior to their infection, increasing to 25 $(60 \%)$ over their entire NICU course. MRSA-infected infants had longer NICU hospitalizations (median 110 days, IQR 601-153 days) than colonized-only infants (median 60 days, IQR 40-99 days, $p<0.001)$. Twenty-three $(55 \%)$ infected infants were colonized at discharge compared to 55 (44\%) colonized-only infants $(p=0.22)$.

\section{Maternal data}

Of 162 mothers overall, the majority (138 [85\%]) were documented to have received antibiotics while admitted prior to childbirth; $12(7 \%)$ did not receive antibiotics and 12 (7\%) have unknown antibiotic use. Nine mothers received antibiotics with activity against MRSA; of these mothers, $1(11 \%)$ of their infants developed symptomatic MRSA infection. Twenty-two mothers (14\%) had a documented history of MRSA infection; 5 (23\%) of these mothers were screened for MRSA colonization upon admission for childbirth and 2 (40\%) were MRSAcolonized. Of these 22 mothers, $5(23 \%)$ of their infants developed symptomatic MRSA infection.

\section{Longitudinal data}

From July 2009 to August 2019 (the end of our data collection period), 46 patients $(36 \%)$ were readmitted to SLCH within 1 year after discharge (Table 2). MRSA-infected infants (18 [53\%]) were more likely to be rehospitalized within 1 year than colonized-only infants (28 [30\%]; $p=$ 0.02). Median days from discharge to readmission did not differ significantly between groups (109 days [IQR 40-239] for MRSA-infected infants, 69 days [IQR 35-167] for colonized-only infants; $p=0.59$ ). One MRSA-infected patient was subsequently admitted for an MRSA abscess; two colonized-only controls (for whom longitudinal data were available) developed MRSA abscesses that did not require hospital admission.

Of 129 infants with longitudinal colonization status available, 16 were colonized with MRSA during a subsequent hospital visit (8 of 34 [24\%] MRSA-infected infants, 8 of 95 [8\%] colonized-only infants; $p=0.03)$. Of 16 infants MRSA-colonized longitudinally, 11 (69\%) were colonized at the time of discharge from their initial hospitalization; 45 (40\%) infants not colonized during follow-up were colonized at the initial discharge $(p=0.03)$. Patients colonized with MRSA longitudinally had longer initial NICU courses (median 124 days, IQR 58-158 days) compared to those who were not colonized longitudinally (median 82 days, IQR 48-115 days, $p=0.04$ ).
Table 2 Subsequent admission diagnoses.

\begin{tabular}{|c|c|}
\hline Category & Specific diagnosis \\
\hline Respiratory diagnoses & $\begin{array}{l}\text { Asthma exacerbation } \\
\text { Pneumonia } \\
\text { Bronchiolitis } \\
\text { Croup } \\
\text { Aspiration pneumonia } \\
\text { Cystic fibrosis exacerbation } \\
\text { Subglottic stenosis after procedure }\end{array}$ \\
\hline Cardiovascular diagnoses & $\begin{array}{l}\text { Arrest after tracheostomy decannulation } \\
\text { Dehydration } \\
\text { Subglottic stenosis }\end{array}$ \\
\hline Infectious diagnoses & $\begin{array}{l}\text { Abscess of left buttocks } \\
\text { Pyelonephritis, renal abscess }\end{array}$ \\
\hline Neurologic diagnoses & $\begin{array}{l}\text { Evaluation with EEG } \\
\text { Febrile seizure }\end{array}$ \\
\hline GI diagnoses & Bloody stools \\
\hline Surgical diagnoses & $\begin{array}{l}\text { Foreign body removal } \\
\text { VP shunt malfunction } \\
\text { Hiatal hernia repair } \\
\text { Cleft palate repair } \\
\text { Laryngotracheal reconstruction } \\
\text { Peritoneal dialysis catheter placement } \\
\text { Dislocated left hip } \\
\text { Inguinal hernia repair } \\
\text { Cochlear implant placement } \\
\text { Closure of ostomy and mucous fistula } \\
\text { Burns to bilateral feet }\end{array}$ \\
\hline Other diagnoses & $\begin{array}{l}\text { Failure to thrive } \\
\text { ALTE (apparent life threatening event) } \\
\text { Near drowning }\end{array}$ \\
\hline
\end{tabular}

In multivariable analysis, patients who required subsequent hospitalization were more likely to have been an MRSA-infected case than a colonized-only control (aOR 2.85, 95\% CI 1.29-6.30) and to have had a longer NICU course (aOR 1.01, 95\% CI 1.00-1.02) during the index hospitalization. Rehospitalized infants were less likely to have received maternal breast milk (aOR 0.22 , 95\% CI 0.07-0.75) during the index hospitalization.

\section{Discussion}

This case-control study informs factors associated with progression of MRSA nasal colonization to development of symptomatic infection in critically-ill neonates. Colonized infants who developed infection became colonized earlier in their NICU course, received fewer days of systemic antibiotics, were less likely to receive mupirocin for 
decolonization, were more likely to receive donor breast milk, and were exposed to longer courses of ventilation prior to their infection. Longitudinally, infected infants had more protracted hospital courses and were more likely to be readmitted to the hospital after discharge. Infected and colonized-only infants had similar colonization prevalence during subsequent hospital visits, though infants who were colonized at the time of NICU discharge (for the index hospitalization) were more likely to be colonized during subsequent admissions. To understand the progression from colonization with MRSA to infection, we must consider routes of MRSA acquisition, portals of entry for the development of symptomatic infection, and protective effects of decolonization.

Neonates acquire MRSA from parents and other caregivers. Several investigations of $S$. aureus and MRSA newborn colonization in the USA have demonstrated that within the mother-infant dyad, vertical transmission is possible, but less likely than horizontal transfer after birth $[17,18]$. Infant colonization with $S$. aureus is high in the first month of life (peaking at 8 weeks) and then tapers by 6 months of age [17, 18]. However, these prior studies evaluated $S$. aureus colonization exclusively in term infants; colonization patterns in preterm infants are not well described. The opportunity for horizontal transfer lies in developmental practices, such as kangaroo care and skin-to-skin contact, encouraged among preterm infants. In our cohort of neonates, only mothers with a history of MRSA infection were tested for MRSA prior to giving birth, precluding our ability to interrogate a correlation between maternal colonization and development of infection in neonates.

Portals of entry likely contribute to development of symptomatic infection from commensal organisms. Infants who developed symptomatic MRSA infection during their NICU course were of lower gestational age and birth weight than those who did not develop infection. These more premature, smaller infants likely have mucosal and skin barriers that are more easily disrupted or immune systems that are even less mature than infants born at later gestational ages and larger birth weights. Most infants in both arms of the cohort had endotracheal tubes, nasogastric/ orogastric tubes, and central vascular lines, signifying that the presence of these devices could predispose infants to colonization. However, infected infants had longer courses of mechanical ventilation, demonstrating that a foreign body placed directly into a colonized site for a prolonged period could predispose these infants to infection. Infants who received donor breast milk were also more likely to develop infection. This may reflect a group of infants who typically received donor breast milk (i.e., younger gestational age and lower birth weight infants) or alteration of antibodies that are typically passed through breast milk through the process of pasteurization [19].
Infants who developed symptomatic infection were less likely to receive the standard decolonization protocol of $2 \%$ intranasal mupirocin twice daily for 7 days following detection of colonization. Decolonization of neonates in the NICU with mupirocin has minimal adverse effects and is efficacious in decolonizing MRSA-colonized infants [11]. Parental decolonization is another strategy that has been recently evaluated in an attempt to limit neonatal MRSA acquisition and subsequent infection. A randomized controlled trial conducted in a NICU in Baltimore demonstrated that decolonization of $S$. aureus-colonized parents resulted in a $57 \%$ reduction in incidence of infant acquisition of a $S$. aureus strain concordant with a parental strain [20, 21]. To reduce infant colonization and infection, a combination of strict adherence to infant decolonization protocols and decolonization of parents may be necessary. Mothers hospitalized in the antepartum unit are another group for whom MRSA surveillance should be considered; detecting and decolonizing these mothers prior to delivery could interrupt subsequent transmission to premature infants.

Development of symptomatic MRSA infection was associated with long-term implications during the remainder of the NICU course and after discharge. Infants who developed infection were exposed to longer courses of both systemic antibiotics and systemic steroids during their overall hospitalization than colonized-only infants, altering the already-fragile infant immune system and gut microbiome [22, 23]. Infected infants were also more likely to undergo at least one surgical procedure during their NICU hospitalization, particularly following their MRSA infection. This is likely attributable to the increase in the severity of clinical illness that results from symptomatic infection. As a result, infected infants also had a longer median length of NICU hospitalization (110 days) than colonized-only infants (60 days). After discharge, infected infants were more likely to have subsequent hospitalizations than colonized-only infants. Most rehospitalizations were secondary to respiratory distress; only one rehospitalization was secondary to reinfection with MRSA (buttock abscess).

This study has several limitations. This was a retrospective study conducted at one tertiary care center and thus the findings may not be generalizable to other geographic areas. During the study period, the SLCH NICU conducted active surveillance for MRSA only; however, the majority of symptomatic $S$. aureus infections were methicillinsusceptible $S$. aureus, limiting sample size of symptomatic infection with MRSA. At last, only a portion of the cohort had follow-up documentation available, and some infants may have been admitted to other hospitals after SLCH discharge; therefore, the longitudinal data may not be a complete reflection of colonization patterns or morbidity associated with MRSA colonization or infection in former NICU infants over time. 


\section{Conclusion}

In conclusion, the development of symptomatic MRSA infection in critically-ill colonized infants is multifactorial. Immature mucous membranes and skin barriers provide potential portals of entry for colonization to develop into symptomatic infection. The development of symptomatic infection is associated with increased morbidity. Reducing colonization via parental surveillance and decolonization in combination with infant decolonization may prevent future infection in already-colonized infants.

Acknowledgements The authors thank Jennifer Wambach, MD and Misty Good, MD, MS for their thoughtful review of this paper.

Funding information This work was supported by the Children's Discovery Institute of Washington University and St. Louis Children's Hospital [to S.A.F], National Institutes of Health/National Institute of Allergy and Infectious Diseases [grant number K23-AI091690 to S.A. F.], the National Center for Advancing Translational Sciences at the National Institutes of Health [Grant Number UL1-TR002345 to S.A. F.], and the Agency for Healthcare Research and Quality [grant numbers R01-HS021736, R01-HS024269 to S.A.F.]. These funding sources had no role in the design and conduct of the study, management, analysis, and interpretation of the data preparation, review, or approval of the paper or decision to submit the paper for publication. The content is solely the responsibility of the authors and does not necessarily represent the official views of the National Institutes of Health or the Agency for Healthcare Research and Quality.

Author contributions Concept and design: CRS, PGH, PJR, MGB, BBW, and SAF. Acquisition, analysis, or interpretation of data: CRS, PGH, PJR, SH, HEW, MGB, RMT, and SAF. Drafting of the paper: CRS, PGH, PJR, SH, HEW, and SAF. Critical revision of the paper for important intellectual content: CRS, PGH, PJR, SH, HEW, MGB, RMT, BBW, and SAF. Statistical analysis: CRS, PGH, SH, HEW, and SAF. Obtained funding: SAF. Administrative, technical, or material support: MGB and RMT. Supervision: PJR, BBW, and SAF.

\section{Compliance with ethical standards}

Conflict of interest The authors declare no competing interests.

Publisher's note Springer Nature remains neutral with regard to jurisdictional claims in published maps and institutional affiliations.

\section{References}

1. Stoll BJ, Hansen N, Fanaroff AA, Wright LL, Carlo WA, Ehrenkranz RA, et al. Late-onset sepsis in very low birth weight neonates: the experience of the NICHD Neonatal Research Network. Pediatrics. 2002;110:285-91.

2. Nelson MU, Gallagher PG. Methicillin-resistant Staphylococcus aureus in the neonatal intensive care unit. Semin Perinatol. 2012;36:424-30.

3. Schultz ED, Tanaka DT, Goldberg RN, Benjamin DK Jr., Smith PB. Effect of methicillin-resistant Staphylococcus aureus colonization in the neonatal intensive care unit on total hospital cost. Infect Control Hosp Epidemiol. 2009;30:383-85.
4. Song X, Perencevich E, Campos J, Short BL, Singh N. Clinical and economic impact of methicillin-resistant Staphylococcus aureus colonization or infection on neonates in intensive care units. Infect Control Hosp Epidemiol. 2010;31:177-82.

5. Huang SS, Platt R. Risk of methicillin-resistant Staphylococcus aureus infection after previous infection or colonization. Clin Infect Dis. 2003;36:281-85.

6. Zervou FN, Zacharioudakis IM, Ziakas PD, Mylonakis E. MRSA colonization and risk of infection in the neonatal and pediatric ICU: a meta-analysis. Pediatrics. 2014;133:e1015-23.

7. Popoola VO, Budd A, Wittig SM, Ross T, Aucott SW, Perl TM, et al. Methicillin-resistant Staphylococcus aureus transmission and infections in a neonatal intensive care unit despite active surveillance cultures and decolonization: challenges for infection prevention. Infect Control Hosp Epidemiol. 2014;35:412-18.

8. Seybold U, Halvosa JS, White N, Voris V, Ray SM, Blumberg HM. Emergence of and risk factors for methicillin-resistant Staphylococcus aureus of community origin in intensive care nurseries. Pediatrics. 2008;122:1039-46.

9. Reich PJ, Boyle MG, Hogan PG, Johnson AJ, Wallace MA, Elward AM, et al. Emergence of community-associated methicillin-resistant Staphylococcus aureus strains in the neonatal intensive care unit: an infection prevention and patient safety challenge. Clin Microbiol Infect. 2016;22:645e1-e8.

10. Carey AJ, Della-Latta P, Huard R, Wu F, Graham PL 3rd, Carp D, et al. Changes in the molecular epidemiological characteristics of methicillin-resistant Staphylococcus aureus in a neonatal intensive care unit. Infect Control Hosp Epidemiol. 2010;31:613-19.

11. Kotloff KL, Shirley DT, Creech CB, Frey SE, Harrison CJ, Staat $\mathrm{M}$, et al. Mupirocin for Staphylococcus aureus decolonization of infants in neonatal intensive care units. Pediatrics. 2019;143: e20181565.

12. Milstone AM, Elward A, Song X, Zerr DM, Orscheln R, Speck K, et al. Daily chlorhexidine bathing to reduce bacteraemia in critically ill children: a multicentre, cluster-randomised, crossover trial. Lancet. 2013;381:1099-106.

13. Huang YC, Chou YH, Su LH, Lien RI, Lin TY. Methicillinresistant Staphylococcus aureus colonization and its association with infection among infants hospitalized in neonatal intensive care units. Pediatrics. 2006;118:469-74.

14. Milstone AM, Goldner BW, Ross T, Shepard JW, Carroll KC, Perl TM. Methicillin-resistant Staphylococcus aureus colonization and risk of subsequent infection in critically ill children: importance of preventing nosocomial methicillin-resistant Staphylococcus aureus transmission. Clin Infect Dis. 2011;53:853-59.

15. Kaushik A, Kest H, Zauk A, DeBari VA, Lamacchia M. Impact of routine methicillin-resistant Staphylococcus aureus (MRSA) surveillance and cohorting on MRSA-related bloodstream infection in neonatal intensive care unit. Am J Perinatol. 2015;32:531-36.

16. Horan TC, Andrus M, Dudeck MA. CDC/NHSN surveillance definition of health care-associated infection and criteria for specific types of infections in the acute care setting. Am J Infect Control. 2008;36:309-32.

17. Jimenez-Truque N, Tedeschi S, Saye EJ, McKenna BD, Langdon W, Wright JP, et al. Relationship between maternal and neonatal Staphylococcus aureus colonization. Pediatrics. 2012;129: e1252-9.

18. Peacock SJ, Justice A, Griffiths D, de Silva GD, Kantzanou MN, Crook D, et al. Determinants of acquisition and carriage of Staphylococcus aureus in infancy. J Clin Microbiol. 2003;41: 5718-25.

19. Demers-Mathieu V, Huston RK, Markell AM, McCulley EA, Martin RL, Spooner M, et al. Differences in maternal immunoglobulins within mother's own breast milk and donor breast milk and across digestion in preterm infants. Nutrients. 2019;11:920. 
20. Milstone AM, Koontz DW, Voskertchian A, Popoola VO, Harrelson $\mathrm{K}$, Ross $\mathrm{T}$, et al. Treating parents to reduce NICU transmission of Staphylococcus aureus (TREAT PARENTS) trial: protocol of a multisite randomised, double-blind, placebocontrolled trial. BMJ Open. 2015;5:e009274.

21. Milstone AM, Voskertchian A, Koontz DW, Khamash DF, Ross T, Aucott SW, et al. Effect of treating parents colonized with Staphylococcus aureus on transmission to neonates in the intensive care unit: a randomized clinical trial. JAMA 2019;323:319-28.
22. Gasparrini AJ, Wang B, Sun X, Kennedy EA, HernandezLeyva A, Ndao IM, et al. Persistent metagenomic signatures of early-life hospitalization and antibiotic treatment in the infant gut microbiota and resistome. Nat Microbiol. 2019;4: 2285-97.

23. Baumann-Dudenhoeffer AM, D'Souza AW, Tarr PI, Warner BB, Dantas G. Infant diet and maternal gestational weight gain predict early metabolic maturation of gut microbiomes. Nat Med. 2018; 24:1822-29. 